\title{
Polyclinics and psychiatry: risks and opportunities
}

\author{
Linda Gask, ${ }^{1}$ Suresh Joseph, ${ }^{2}$ Michele Hampson ${ }^{3}$
}

The Psychiatrist (2010), 34, 106-109, doi: 10.1192/pb.bp.108.023002

'University of Manchester:

${ }^{2}$ Northumberland, Tyne and Wear NHS

Trust; ${ }^{3}$ Nottinghamshire Healthcare

NHS Trust

Correspondence to Linda Gask

(Linda.Gask@manchester.ac.uk)
Summary The arrival of the 'polyclinic' or 'GP-led health centre' has been signalled in the review of the National Health Service. A variety of options have been proposed for the way in which polyclinics will incorporate specialist services to work alongside primary care, and the relevance of these models to mental healthcare is considered. Polyclinics provide new opportunities but with those possibilities come potential threats and risks. Of key importance is the threat that they will re-institutionalise mental healthcare after many years of breaking down such barriers. Buildings provide shared space, but new working practices are more difficult to achieve.

Declaration of interest None.

\section{Polyclinics and psychiatry}

\section{What is a polyclinic? A European perspective}

The preliminary review of the National Health Service (NHS) in England, led by Lord Darzi, proposed the development of 'polyclinics', ${ }^{1}$ which in the final report are referred to as 'GP-led health centres'. ${ }^{2}$ Although there is considerable confusion in the literature about what it actually means, ${ }^{3}$ the term polyclinic will be familiar to those who have worked in the former Soviet countries of Europe (e.g. Russia and the Ukraine), where the institution called a polyclinic was essentially an out-patient unit staffed by specialists supported by lower-status doctors. Attempts in recent health reforms in those countries to introduce a tier of vocationally trained general practitioners (GPs) into such settings were met with some resistance, not least from specialists. ${ }^{4,5}$ However, polyclinics, once limited to the old East Germany, are now opening across Germany ${ }^{6}$ and this newer model of polyclinic, promoted within the UK as early as $1920{ }^{7}$ brings together primary care, specialists, diagnostic and therapeutic services under one roof, thus creating a large, extended, multiprofessional, out-patient clinic.

\section{Risks}

Bringing specialists and generalists together in community settings seems to offer an attractive model for improving patient care and minimising unnecessary out-patient care. However, there are a number of issues to resolve about these new centres, including the extent to which they will replace or supplement existing primary care services, ${ }^{8}$ the nature and level of expertise of the specialist workers who will be deployed into them, and how they will interface with ongoing developments in care pathways for people with mental health problems in the community. In some countries in Europe where specialists are based outside the hospital, patients still have the choice of consulting the specialist first without the GP playing a gatekeeper role (although this is beginning to change in Germany and France). This is not the Darzi vision of the polyclinic, which will be clearly 'GP-led'.

\section{Other developments}

Indeed, GP-led health centres or polyclinics should be seen as only one possible method for the delivery of the broader 'Darzi' aims: increasing the delivery of evidence-based care, increasing access to specialist services in settings other than traditional hospital ones, and increasing clinical engagement in the process of service delivery. Their ability to achieve these aims will depend on the models adopted, the opportunity to adopt a flexible, incremental approach where necessary, the involvement of the practitioners and their willingness to embrace change and adequate resources. The involvement of service users, families and carers in the polyclinic design will be crucial to their success. Psychiatry has been at the forefront of developing community-based services and has led on to the integration of health and social care. We would therefore wish to see new centres designed not simply as an extended out-patient model but as an opportunity to create further service integration and provide opportunities for wider engagement in community activities. Some new primary care resource centres in cities, for example, have provided places not only for access to health and social care but also for social interaction and further education. Size is key, as there is a risk that large clinics may become mini-hospitals and 'institutional', which will defeat their purpose.

\section{Working towards greater inclusion}

The most helpful approach would facilitate the integration of a range of specialist mental health inputs into primary care. Few people receive specialist mental healthcare; in some parts of the country, specialist services are in regular contact with only $1-2 \%$ of the population. ${ }^{9}$ This is likely to be those with severe and enduring mental illness and there 
is evidently little or no influence from mental health specialists on the care of the large number of individuals who still have very significant mental health needs. These include people in the general hospital sector (out-patient and in-patient), residential care settings (statutory and voluntary sector, includes elderly care facilities and community provision for people with intellectual disabilities), as well as people in general community settings. There have been experiments with several models of care delivery. Even the more successful models (general psychiatry, specialist community teams and liaison psychiatry models) have a very narrow focus and are variable in their impact. There is an imperative, supported by primary care staff, to address this service gap ${ }^{10}$ for people with serious but non-psychotic mental disorder and to consider how mental health services can extend the provision of their expertise to a larger number of people without becoming further overburdened.

\section{Inevitable changes}

Any future solution will require both structural and functional change, and critically, a need for behavioural change on the part of practitioners, GPs, other specialists, healthcare professionals and psychiatrists alike. This has been notoriously difficult to achieve, even in areas that have nurtured these models. ${ }^{11}$ We therefore recommend a stepby-step approach to implementation. These are inherently not approaches that can be 'rolled out' in the usual way in which such initiatives are intended to be delivered by policy makers.

New models of care should thus enable mental health expertise to be projected into areas where it is lacking: community settings, general hospital settings, social care settings, and both residential and day service provision.

\section{Different models}

A recent review of the evidence base for shifting specialist care from hospitals to the community ${ }^{12}$ considered the risks and potential benefits of three models of working that are relevant to mental health input into a polyclinic:

1. substitution of services by transferring to new practitioners, who in psychiatry would be practitioners with a special interest in mental health or other non-medical therapists

2. shifting services: either psychiatric out-patient clinics into primary care settings with no change of personnel, or basing community mental health teams (CMHTs) in the local polyclinic

3. joint working between:

a. other hospital specialists and psychiatrists (e.g. paediatricians and child psychiatrists)

b. different specialties within psychiatry (e.g. adult and child, adult and liaison, adult and older adults)

c. primary and specialist care in order to develop new roles for consultants in 'primary care psychiatry' and to promote better links with liaison psychiatry and primary care.
There is also a developing evidence base on ways of working at the interface between primary and specialist mental healthcare, ${ }^{13,14}$ which, although it does not include any research into polyclinic models per se, can inform our thinking on how they might or might not work effectively.

These models are considered in turn below.

\section{New practitioners}

Practitioners with a special interest in mental health

One option would be for polyclinics to be a focus for mental health delivery from new practitioners with a special interest in mental health, and this would seem a likely development for primary care trusts to consider if consultant psychiatrists choose not to be based there. The development of specific educational competencies and standards for such practitioners, who may include GPs and also pharmacists, is still at a rudimentary stage (despite development of an accreditation process by the Department of Health; www.pcc.nhs.uk/173.php), with more attention still required on specific training issues and ongoing supervision and mentorship from mental health specialists. There is a risk not only of a lack of cost-effectiveness if assumptions are made that practitioners with a special interest in mental health can substitute for consultants without adequate training and supervision, but also of risks to patients. ${ }^{15}$

\section{Nurses and psychologists}

Other specialist mental health practitioners, such as nurses and psychologists, have been based in primary care settings over the past 25 years, and their effectiveness varies dependent on the models of care utilised. The effectiveness of mental health nurses in this setting, in the management of common disorders such as anxiety and depression when utilising generic ${ }^{16}$ or problem-solving models of care ${ }^{17}$ has not been demonstrated to be superior to usual GP care. The traditional 'attached community psychiatric nurse' model of the past, although beloved of GPs, should not be replicated in its original form. Closer working of psychological therapy services with primary care will be nationally implemented through the Improving Access to Psychological Therapies (IAPT) initiative and these services should have a presence in the polyclinic.

\section{Shifting mental health services into the polyclinic}

There are three options: moving CMHTs into the polyclinic, moving clinic sessions into the polyclinic and/or opening up new clinics for direct referral from GPs to consultants in the clinic.

\section{Moving CMHTs into the polyclinic}

Shifting a team base into the polyclinic will allow much better linking between primary and secondary care practitioners and would reduce the cost of running a team base alongside the polyclinic, potentially save costs in outof-hours working and staff travelling, but would have the added risk of increasing the institutional feeling that CMHT bases have successfully diminished in many community bases. Attention would need to be given to the physical environment to ensure a range of room sizes for individual, 
family and group meetings, shared clinic rooms for physical examinations, depot medication and access to phlebotomy services, sufficient access to personal computers and adequate desk space, and finally attention to staff safety in the design. However, and most important of all, not only should there be a shared space for clinicians to meet, facilitate communication and mutual respect, but also there should be no stigmatisation of mental health service users, for example by giving them separate waiting areas.

\section{Psychiatric out-patient clinics in primary care settings}

Locating existing out-patient clinics in primary care without adding other ingredients to the model will improve access to underserved populations and patient satisfaction (in terms of potentially reduced stigma attending a polyclinic rather than a psychiatric out-patient department in a mental health unit, and ease of access) ${ }^{13}$ However, this is less efficient not only in terms of staff travelling time but also in utilising time effectively related to patient nonattendance. $^{12}$ In its own right it also does not improve communication with primary care staff. Consultants in general psychiatry have operated such clinics in peripheral parts of their 'patch' for many years. The link-working role with primary care recommended in the National Service Framework for mental health ${ }^{18}$ has not been widely implemented. One option for polyclinics would be the shift of some mental health worker contacts to this setting from CMHTs, with the possibility of better care coordination of physical healthcare for clients on enhanced care programme approach (CPA) and greater involvement of GPs in CPA meetings.

The risk of opening up consultant sessions to new referrals from GPs in a polyclinic is that these will be patients who do not necessarily require specialist input and might be more cost-effectively seen by another mental health worker (e.g. people with adjustment disorders). The challenge would be to ensure this would not happen, which would require a form of joint working (see below).

Of relevance to both of these models, there is no evidence that locating specialist mental health workers in the primary care setting has any impact on GPs' knowledge or skills. ${ }^{19}$

\section{Shared care models}

Mental health has been at the forefront of developing shared care models with primary care, although these have not been widely implemented. General practitioners are likely to want help with their patients presenting with common non-psychotic disorders who currently often fail to meet criteria for access to CMHT services. There are various ways of approaching this which may be relevant to services provided in a polyclinic.

The polyclinic might provide a focus for closer joint working between psychiatrists and other medical specialists, for example, between child psychiatry and community paediatricians, as well as an opportunity for more joinedup working across interfaces in mental healthcare such as between child and adult services in the provision of services for young people.

In terms of closer working with primary care, the consultation-liaison model ${ }^{13}$ in which the psychiatrist sees the patient only after discussion with the GP, meets regularly to discuss cases and discharges the patient back to GP care earlier than in routine care, is an option for the polyclinic specialist sessions, but will require a revision of the usual working practices of both specialists and GPs and has not been shown to be beneficial in terms of improving clinical outcomes. ${ }^{19}$ General practitioners want to talk directly to a psychiatrist and get an expert opinion, ${ }^{20}$ something which, in the new era of single point of access and fragmentation of general psychiatry, has proved increasingly difficult for them. It is also questionable where a consultation-liaison type of clinic is the most effective use of specialist time, as many of the people so referred might be more effectively managed by other mental health workers in the community (such as graduate mental health workers and gateway workers) with support and supervision from a psychiatrist.

The model of shared care incorporates elements of collaborative care and stepped care, with the specialist being utilised to support and supervise at lower levels of severity, and consult only where a specialist opinion is required. A systematic review of the evidence for this model and the specific role of the specialist within it in the case of common chronic disorders are both available, ${ }^{21,22}$ and the research evidence would favour this approach.

Specialist input to the polyclinic for people with common mental health problems could therefore occur as an integral part of a new type of shared care service for common mental health problems, within a primary care team providing the focus for steps 2 and 3 of IAPT (www.iapt.nhs.uk) and utilising a stepped, collaborative care model delivering both low-intensity psychological therapies and medication management, where required. There is a clear role for a psychiatrist, GP with a special interest and high-intensity psychological therapist in providing supervision, support and consultation to this team. The focus for this team's work will be the polyclinic, where team members, including the psychiatrist, would consult with patients and professionals.

The collaborative care approach offers the best hope of integrating specialist and primary care, including potentially the integration of several specialties, particularly liaison psychiatry. This specialty has much to offer primary care in the development of new collaborative care pathways for people with unexplained somatic symptoms and comorbid mental health problems in other common chronic diseases such as diabetes and coronary heart disease. The chronic disease model/chronic illness care approach ${ }^{23}$ is helpful in this regard and there is much to be gained by making common cause between different specialties in psychiatry, especially liaison and general psychiatrists.

\section{Conclusion}

Much of what we have described above could be achieved without the development of a new building. Buildings provide shared space, but new working practices are more difficult to achieve.

We agree that a demonstration of the clinical outcomes and cost-effectiveness and acceptability, to both service users and professionals, of new GP-led health centres or 
polyclinics, whatever they are finally named, should be among the early activities of implementation, and that by building on existing clinic plans and developments this could be done without delay.

The government should provide incentives and support for the exploration of a range of new models of service provision, including new health centres. These models should address locations, behavioural change issues and the integration through stepped care pathways with hospital-based care, and teams providing more specialist expertise. Examples of such approaches could include: the provision of psychiatric expertise for atypical/somatising patients and those presenting frequently to general hospitals; the development of community models within or alongside existing specialist teams, for example in the early detection and intervention in dementia or depression in older people; enhancing access to elderly psychiatry expertise in community settings, again with a view to preventing relapse/deterioration and prolonged hospital stays; and, last but not least, improving access to mental healthcare in prison.

The College recognises the need for its members to be willing to change their role in relation to the primary care interface and we would wish them to have a key leadership role in this process.

\section{Acknowledgements}

Thanks to Lisetta Lovett for her very helpful comments on an earlier version of the manuscript.

\section{About the authors}

Linda Gask is Professor of Primary Care Psychiatry at the University of Manchester, National Primary Care Research and Development Centre, Suresh Joseph is Acting Medical Director, Northumberland, Tyne and Wear NHS Trust, and Michele Hampson is Consultant in Adult Psychiatry, Nottinghamshire Healthcare NHS Trust.

\section{References}

1 Imison C, Naylor C, Maybin J. Under One Roof: Will Polyclinics Deliver Integrated Care? King's Fund, 2008.

2 Department of Health. High Quality Care for All: NHS Next Stage Review Final Report. Department of Health, 2008.

3 Finch R. When is a polyclinic not a polyclinic? BMJ 2008; 336: 916-8.

4 Rese A, Balabanova D, Danishevski K, McKee M, Sheaff R. Implementing general practice in Russia: getting beyond the first steps. BMJ 2005; 331: 204-7.

5 Healy J, McKee M. Health sector reform in central and eastern Europe: the professional dimension. Health Policy Plan 1997; 2: 286-95.
6 NHS Evidence - Health Management. Polyclinics. NHS Evidence, 2009 (http://www.library.nhs.uk/HealthManagement/ViewResource.aspx? resID=267332)

7 Ministry of Health, Consultative Council on Medical and Allied Services. Interim Report on the Future of Medical and Allied Services. HMSO, 1920 (http://www.sochealth.co.uk/history/Dawson.htm).

8 Alford S. Polyclinics to replace traditional doctors' surgeries. Sunday Times, 2008; 16 February (http://www.timesonline.co.uk/tol/news/uk/ health/article3380852.ece).

9 The Health and Social Care Information Centre. Mental Health Bulletin: Second Report on Experimental Statistics from Mental Health Minimum Dataset (MHMDS) Annual Returns, 2003-2008. The Health and Social Care Information Centre, 2009.

10 Boardman J, Henshaw C, Willmott S. Needs for mental health treatment among general practice attenders. Br J Psychiatry 2004; 185: 318-27.

11 Kilbourne AM, Schulberg HC, Post EP, Rollman BL, Belnap BH, Pincus $\mathrm{HA}$. Translating evidence-based depression management services to community-based primary care practices. Milbank Q 2004; 82: 631-59.

12 Sibbald B, MacDonald R, Roland M. Shifting care from hospitals to the community: a review of the evidence on quality and efficiency. J Health Serv Res Policy 2007; 12: 110-7.

13 Gask L, Sibbald B, Creed F. Evaluating models of working at the interface between mental health services and primary care. Br J Psychiatry 1997; 170: 6-11.

14 Bower P, Gilbody S. Managing common mental health disorders in primary care: conceptual models and evidence base. BMJ 2005; 330 839-42.

15 Leese B, Bohan M, Gemmell I, Hinder S, Mead N, Pickard S, et al Evaluation of 'Closer to Home' Demonstration Sites: Final Report. National Primary Care Research and Development Centre, 2007.

16 Gournay K, Brooking J. Community psychiatric nurses in primary health care. Br J Psychiatry 1994; 165: 231-8.

17 Kendrick T, Simons L, Mynors-Wallis L, Gray A, Lathlean J, Pickering R, et al. Cost-effectiveness of referral for generic care or problem-solving treatment from community mental health nurses, compared with usual general practitioner care for common mental disorders. Randomised controlled trial. Br J Psychiatry 2006; 189: 50-9.

18 Department of Health. National Service Framework for Mental Health Modern Standards and Service Models. Department of Health, 1999.

19 Bower P, Sibbald B. Systematic review of the effect of on-site mental health professionals on the clinical behaviour of general practitioners. BMJ 2000; 320: 614-7.

20 Chew-Graham C, Slade M, Montâna C, Stewart M, Gask L. Loss of doctor-to-doctor communication: lessons from the reconfiguration of mental health services in England. J Health Serv Res Policy 2008; 13: 6-12.

21 Gilbody S, Bower P, Fletcher J, Richards DA, Sutton A. Collaborative care for depression: a systematic review and cumulative meta-analysis. Arch Intern Med 2006; 166: 2314-21.

22 Gask L. Role of specialists in common chronic diseases. BMJ 2005; 330 651-3.

23 Richards DA, Lovell K, Gilbody S, Gask L, Torgerson D, Barkham M, et al. Collaborative care for depression in UK primary care: a randomised controlled trial. Psychol Med 2008; 38: 279-87. 